OPEN ACCESS

Edited by:

Carlos B. Duarte,

University of Coimbra, Portugal

Reviewed by:

Mohamed Chahine,

Laval University, Canada

Maurizio Taglialatela,

University of Naples Federico II, Italy

*Correspondence: Wei-Ping Liao wpliao@163.net

${ }^{\dagger}$ These authors have contributed equally to this work

Specialty section:

This article was submitted to Molecular Signalling and Pathways, a section of the journal Frontiers in Molecular Neuroscience

Received: 19 October 2021 Accepted: 15 December 2021 Published: 10 January 2022

Citation:

Su T, Chen M-L, Liu L-H, Meng H,

Tang B, Liu X-R and Liao W-P (2022) Critical Role of E1623 Residue in S3-S4 Loop of Nav1.1 Channel and Correlation Between Nature of Substitution and Functional Alteration.

Front. Mol. Neurosci. 14:797628. doi: 10.3389/fnmol.2021.797628

\section{Critical Role of E1623 Residue in S3-S4 Loop of Nav1.1 Channel and Correlation Between Nature of Substitution and Functional Alteration}

\author{
Tao Su ${ }^{1,2 \dagger}$, Meng-Long Chen ${ }^{1,2 \dagger}$, Li-Hong Liu ${ }^{1,2}$, Hen Meng ${ }^{1,2}$, Bin Tang ${ }^{1,2}$, \\ Xiao-Rong Liu ${ }^{1,2}$ and Wei-Ping Liao ${ }^{1,2 *}$
}

'Department of Neurology, Institute of Neuroscience, Second Affiliated Hospital of Guangzhou Medical University, Guangzhou, China, ${ }^{2}$ Key Laboratory of Neurogenetics and Channelopathies of the Ministry of Education of China, Guangzhou, China

Objective: An overwhelming majority of the genetic variants associated with genetic disorders are missense. The association between the nature of substitution and the functional alteration, which is critical in determining the pathogenicity of variants, remains largely unknown. With a novel missense variant (E1623A) identified from two epileptic cases, which occurs in the extracellular S3-S4 loop of $\mathrm{Na}_{v} 1.1$, we studied functional changes of all latent mutations at residue E1623, aiming to understand the relationship between substitution nature and functional alteration.

Methods: Six latent mutants with amino acid substitutions at E1623 were generated, followed by measurements of their electrophysiological alterations. Different computational analyses were used to parameterize the residue alterations.

Results: Structural modeling indicated that the E1623 was located in the peripheral region far from the central pore, and contributed to the tight turn of the S3-S4 loop. The E1623 residue exhibited low functional tolerance to the substitutions with the most remarkable loss-of-function found in E1623A, including reduced current density, less steady-state availability of activation and inactivation, and slower recovery from fast inactivation. Correlation analysis between electrophysiological parameters and the parameterized physicochemical properties of different residues suggested that hydrophilicity of side-chain at E1623 might be a crucial contributor for voltage-dependent kinetics. However, none of the established algorithms on the physicochemical variations of residues could well predict changes in the channel conductance property indicated by peak current density.

Significance: The results established the important role of the extracellular S3-S4 loop in $\mathrm{Na}_{\mathrm{v}} 1.1$ channel gating and proposed a possible effect of local conformational loop flexibility on channel conductance and kinetics. Site-specific knowledge of protein will be a fundamental task for future bioinformatics.

Keywords: missense, sodium channel, SCN1A, epilepsy, prediction 


\section{INTRODUCTION}

The distinct function of a protein depends on the well-organized composition of the amino acids and intricate folding of the molecule. Gene mutations result in either gross protein malformation (referred to destructive variants), that mostly led to haploinsufficiency, such as truncation and splice-site (He et al., 2019), or residue substitution (missense). A part of genetic defects may translate into functional alterations and subsequently lead to human diseases. Advances in genetic sequencing technology have enabled the detection of numerous sequence variants in human beings. An overwhelming majority of the variants are missense (gnomAD, HGMD), which result in the substitution of the amino acid (AA) at residues. In contrast to destructive mutations that result in remarkably damaging effects on protein composition, the functional consequence of missense mutations is far more unpredictable. Our previous study demonstrated that the molecular sub-regional location of the variant plays a critical in determining the damaging effect of the mutations (Tang et al., 2020). However, the pathogenicity or functional impact of a missense mutation depends not only on its location but also potentially on the nature of amino acid substituted, such as molecular mass, polarity, and acidity. The association between the nature of substitution and the functional alteration, which remains largely unknown, is a critical aspect in exploring the molecular mechanism underlying the pathogenicity of variants.

Genetic defects in voltage-gated sodium channel $\left(\mathrm{Na}_{\mathrm{v}} 1.1\right) \alpha$ subunit encoded by $S C N 1 A$ are a major cause of epilepsy. To date, more than $1,700 S C N 1 A$ variants have been reported to be associated with epilepsy and other episodic disorders ${ }^{1}$ (Meng et al., 2015). The SCN1A associated epilepsies compose a wide spectrum of phenotype ranging from milder febrile seizures to severe epileptic conditions, typically Dravet syndrome (DS; Claes et al., 2003; Nabbout et al., 2003; Ceulemans et al., 2004; Mulley et al., 2005; Harkin et al., 2007; Gambardella and Marini, 2009; Dravet and Oguni, 2013; Kasperaviciute et al., 2013; Gataullina and Dulac, 2017). Previous studies have demonstrated that DS or severe epilepsies are associated with destructive variants such as nonsense and frameshift variants, or missense in the pore-region that caused loss-of-function of $\mathrm{Na}_{\mathrm{v}}$ 1.1. Although missense variants in other regions of $\mathrm{Na}_{\mathrm{v}} 1.1$ have also been reported (Meng et al., 2015), their functional relevance has not been well studied. Factors that determine the pathogenicity of SCN1A missense variants remain unclear.

In this study, we identified a novel distinct missense SCN1A variant (c.4868A $>$ C/E1613A) in two cases with epilepsy and febrile seizures. The substitution occurs at the putative extracellular loop linking S3 and S4 in DIV. The functional role of the extracellular S3-S4 loop remains largely unknown, except that a few studies identified toxin binding sites within the loops (Rogers et al., 1996; Bosmans et al., 2008; Wang et al., 2011). To dig deep into the functional role of E1623 and the factors that influence the pathogenicity of missense variants, six E1623 mutants with all possible

\footnotetext{
${ }^{1}$ http://scnla.caae.org.cn/
}

substitutions caused by single nucleotide variations (SNV) were artificially created using site-directed mutagenesis. We determined the electrophysiological properties of these mutants and analyzed the correlations between the residue properties and electrophysiological alterations.

\section{MATERIALS AND METHODS}

\section{Patient and Genetic Analysis}

Diagnoses and treatments of the patients were conducted in the epilepsy center of the second affiliated hospital of Guangzhou Medical University. Clinical data including medical records, standardized questionnaires, and EEG recordings were collected. The probands and relevant familial members were assessed for genetic variations using a standardized protocol after providing written informed consent. This study was approved by the Research Ethics Board of the Hospital. Genomic DNAs were prepared from ethylene diaminetetraacetic acid-treated whole blood samples. The SCN1A mutation was identified by direct SCN1A screening as in our previous report (Liao et al., 2010). The SCN1A mutations were described according to the nomenclature established (den Dunnen and Antonarakis, 2000), and numbering was started from the initiating ATG codon.

\section{Bioinformatics}

$\mathrm{Na}_{\mathrm{v}}$ channel nucleotide sequences were derived from the NCBI database ${ }^{2}$. The NCBI database was queried with amino acid sequences of human $\mathrm{Na}_{\mathrm{v}} 1.1$ to obtain the corresponding information of DNA locus and related functional regions. To determine whether other amino acid substitutions occur in the vicinity of the mutation site, information of SNPs and mutations were queried from the SNP database of the NCBI and our SCN1A mutation database ${ }^{1}$. All amino acid sequences of the $\mathrm{Na}_{\mathrm{v}}$ families were retrieved from GenBank and saved as individual FASTA formatted files. The sequences including the S3-S4 region of DIV were subjected to multiple sequence alignment analysis by Clustal Omega with a few modifications in the color grouping. The sequence ranges of specific S3-S4 regions are in accord with that is annotated for human $\mathrm{Na}_{\mathrm{v}} 1.1$ isoform 1 in the NCBI database.

The high resolution cryogenic electron microscopy structure of human $\mathrm{Na}_{\mathrm{v}}$ 1.1 (PDB ID: 7DTD, $3.3 \AA$ taken from the protein data bank $^{3}$ ) was used as a template for subsequent modeling (Pan et al., 2021). Three-dimensional (3D) modeling of the human wild-type (WT) and mutant $\mathrm{Na}_{\mathrm{v}} 1.1$ was performed using SWISSMODEL, an automated homology modeling program ${ }^{4}$.

The predicted effects of all latent amino acid substitutions at E1623 were scored by different established predictive tools based on different substitution score systems, including SIFT, Mutationassessor, PolyPhen, PROVEAN, I-MUTANT suite, SNAP2, and STRUM (Tang et al., 2020).

\footnotetext{
${ }^{2}$ http://www.ncbi.nlm.nih.gov/

${ }^{3}$ https://www.rcsb.org/structure/7DTD

${ }^{4} \mathrm{http}: / /$ swissmodel.expasy.org
} 


\section{Mutagenesis and Heterologous Expression}

Plasmids containing WT full-length sequence of human $\mathrm{Na}_{\mathrm{v}} 1.1$ alpha subunit (pCMV-SCN1A) and beta subunit 2 (pGFP-IRES-SCN2B) was kindly provided by professor Alfred L Jr George (Lossin et al., 2003). A full-length sequence of beta subunit 1 was constructed into a vector driving the bicistronic expression of red fluorescent protein (pDsred-IRES$S C N 1 B$ ) in our lab. Site-directed mutagenesis at E1623 was performed using the Quick Change XL site-directed mutagenesis kit (Stratagene, La Jolla, CA) according to the manufacturer's protocols. All mutations were confirmed by DNA sequencing of the region surrounding the mutation. HEK 293T cells were grown in 1:1 Ham's F-12 and Dulbecco's modified eagle's medium (DMEM) supplemented with $10 \%$ fetal bovine serum, $100 \mathrm{U} / \mathrm{ml}$ of penicillin, and $100 \mu \mathrm{g} / \mathrm{ml}$ streptomycin. The cells were maintained in a humidified incubator at $37^{\circ} \mathrm{C}$ with $5 \% \mathrm{CO}_{2}$. Cells were then co-transfected with pCMV-SCN1A, and pDsredIRES-SCN1B and pGFP-IRES-SCN2B plasmids ( $5 \mu \mathrm{g}$ each) using Lipofectamine 3000 reagent Kit from Thermo Fisher Scientific. After incubation for $12-15 \mathrm{~h}$, cells were replated in $35-\mathrm{mm}$ culture dishes.

\section{Electrophysiological Recordings and Analysis}

Electrophysiological studies were performed $20-48 \mathrm{~h}$ after transfection, according to our previous report (Chen et al., 2015). Channel activity was recorded by using the conventional patch clamp technique in the whole-cell configuration with an Axopatch 200B patch-clamp amplifier (Axon Instruments). The extracellular control solution contained: $140 \mathrm{mM} \mathrm{NaCl}, 3 \mathrm{mM}$ $\mathrm{KCl}, 1 \mathrm{mM} \mathrm{CaCl} 2,1 \mathrm{mM} \mathrm{MgCl}_{2}, 10 \mathrm{mM}$ HEPES, and $5 \mathrm{mM}$ glucose, adjusted to $\mathrm{pH} 7.4,310 \pm 6 \mathrm{mOsm}$. The whole-cell pipette solution contained: $140 \mathrm{mM} \mathrm{CsF}, 1 \mathrm{mM}$ EGTA, $10 \mathrm{mM}$ HEPES, and $10 \mathrm{mM} \mathrm{NaCl}, \mathrm{pH}$ 7.4, adjusted with $\mathrm{CsOH}$, $310 \pm 6 \mathrm{mOsm}$. Cell capacitance was calculated by integrating the area under an uncompensated capacity transient elicited by a 10-mV hyperpolarizing test pulse from a holding potential of $-80 \mathrm{mV}$. Series resistance was compensated by at least $70 \%$ in all recordings. Leakage currents were subtracted by the $\mathrm{P} / \mathrm{N}$ method. The pCLAMP 10.2 software (Axon Instruments) was used for the acquisition and analysis of currents. Current amplitude in response to each test pulse was normalized to the maximum. The voltage dependence of activation and inactivation was determined using standard protocols. The conductance $(G)$ was calculated according to: $G=I_{\text {peak }} /\left(V_{\text {test }}-V_{\text {rev }}\right)$, where $V_{\text {rev }}$ is the $\mathrm{Na}^{+}$reversal potential, $V_{\text {test }}$ is the command potential, and $I_{\text {peak }}$ is the peak current amplitude. $G / G_{\max }$ was then fitted with the following Boltzmann equation: $G / G_{\max }=(1+$ $\left.\exp \left(\left(V_{m}-V_{1 / 2}\right) / k\right)\right)^{-1}$, where $G_{\max }$ is the maximal conductance, $\mathrm{V}_{1 / 2}$ is the half-activation potential, $\mathrm{V}_{\mathrm{m}}$ is the test voltage, and $\mathrm{k}$ is the slope factor. From this equation, we derived parameters of the midpoint $\left(\mathrm{V}_{1 / 2}\right)$ and slope factor of steady-state activation and inactivation curves. Parameters for recovery of channels from fast inactivation were determined using a double pulse protocol. Channels were inactivated with $-10 \mathrm{mV}$ conditioning pulse for $100 \mathrm{~ms}$, followed by command hyperpolarization for varying durations as a recovery period ( $1 \mathrm{~ms}$ to $1 \mathrm{~s}$ ), and subsequent test pulse to $-10 \mathrm{mV}$ (for $10 \mathrm{~ms}$ ). The normalized recovery curve was fit with a double-exponential function to obtain tau for recovery: $\mathrm{I} / \mathrm{I}_{\max }=\mathrm{A} 1 \times(1-\exp (-\mathrm{t} / \tau$-fast $))+\mathrm{A} 2 \times(1-\exp (-\mathrm{t} / \tau$-slow $))$, where $\mathrm{I}$ is the current amplitude at the time point $t$ after the onset of the voltage command, $\mathrm{I}_{\max }$ is the maximal recovery current amplitude, and $\mathrm{A}$ is the amplitude contribution of the different recovery time constants. Time constants of recovery $\tau$-fast and $\tau$-slow were extracted from the equation as recovery parameters.

\section{Statistical Analysis}

Data reported throughout the text and figures are presented as means \pm SEM. Statistical analyses were conducted in SPSS 19.0, using Student's $t$-test when comparisons were made between two groups or by one-way ANOVA with a post hoc Tukey HSD test for comparing data from multiple groups. Significance was assigned at $P<0.05$.

\section{RESULTS}

\section{Epilepsy-Associated Variant E1623A and the Extracellular Loop}

An SCN1A variant c.4868A>C/p.E1623A was identified from two cases of epilepsy, including a case with de novo variant and a familial case with three individuals affected (Figures 1 A-C). Case 1 was a 17.5-year-old boy with Dravet syndrome. The proband of case 2 was diagnosed as epilepsy with febrile seizure plus. The proband's mother and elder sister had several febrile seizures before 6 years old (clinical information refers to Supplementary Data). The variant occurs in exon 26 of SCN1A and leads to the substitution of glutamic acid by alanine at E1623 that locates in the extracellular loop linking S3-S4 in DIV of $\mathrm{Na}_{\mathrm{v}} 1.1$ (Figures 1D,E). The E1623A is a novel missense variant despite several disease-associated or low-frequency variants flanking the site of E1623. The 3D structural modeling showed that E1623 residue and its correlated loop were located on the top of the lateral voltage-sensor domain (VSD, S4; Figures 1F,G).

Sequence alignments for conservation showed that the amino acid sequence of the S3-S4 loop in DIV of $\mathrm{Na}_{\mathrm{v}} 1.1$ is highly conserved across species. It is noteworthy that a potential motif constituted by two residues at positions E1623 and E1626 remains evolutionarily conserved in their negative charged property (Figure 2A). Residues from E1623 to E1626 form a tight turn of the loop on the top of the lateral VSD (Figure 1G). Among different alpha subunits of $\mathrm{Na}_{\mathrm{v}}$ family that contribute to different $\mathrm{Na}_{\mathrm{v}}$ properties, the sequence is slightly variable, but conserved with two alternative acidic residues, glutamate or aspartate, at the equivalent position as E1623 in $\mathrm{Na}_{\mathrm{v}} 1.1$ (Figure 2B). The other three asymmetric domains (DI-III) of human $\mathrm{Na}_{\mathrm{v}} 1.1$ also showed conservation of the glutamate residue, even though they have the much shorter S3-S4 loop (Figures 2C,D).

\section{Consequences of Substitutions at E1623}

To understand the role of this potentially important glutamate residue on channel function and the link between residue property and functional alteration, we generated all the latent substitutions at residue E1623 caused by SNVs. The substitutions 
A

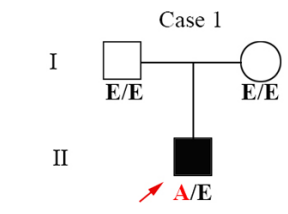

B

$C G A$ G C $T$

Proband

Father

C

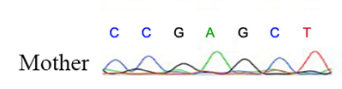

Sister

D

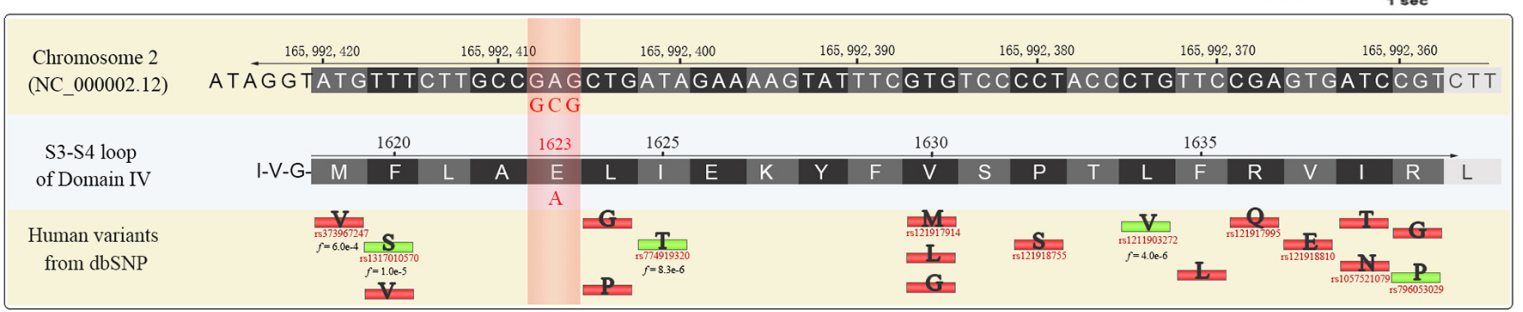

E

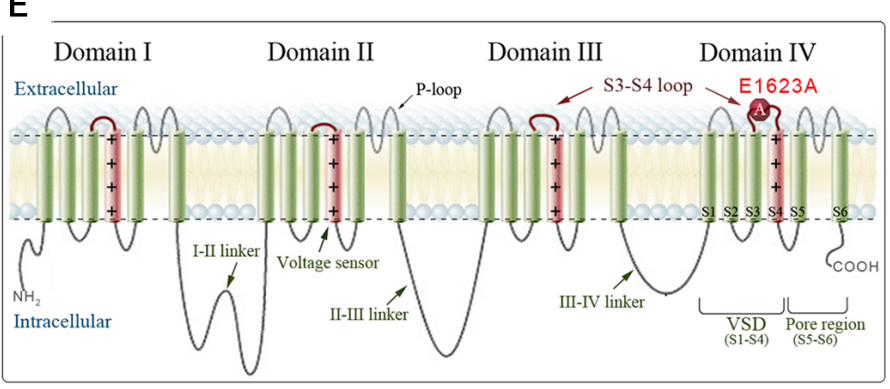

C
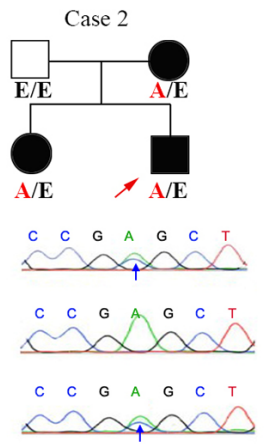

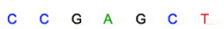

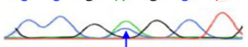

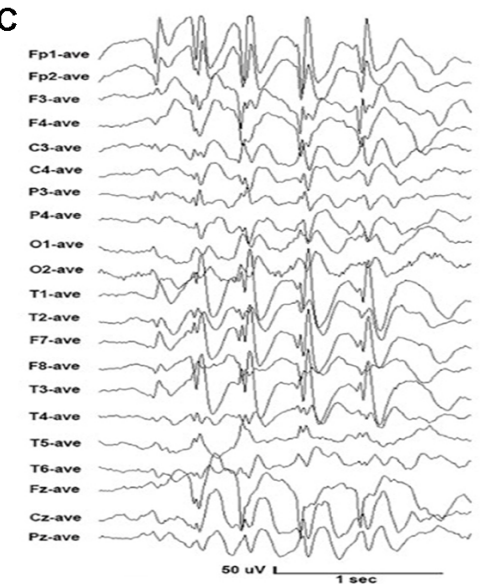

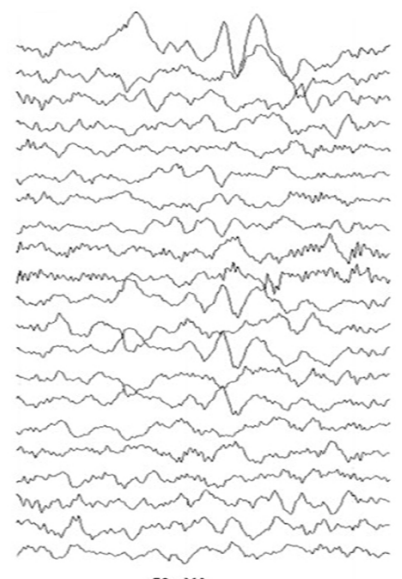

50 uv

F

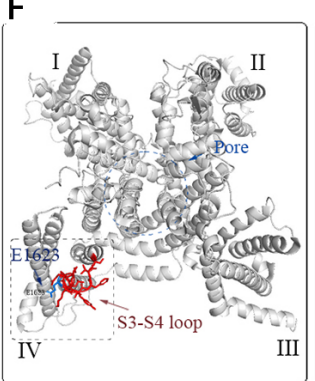

G

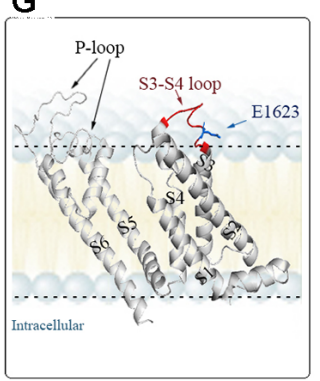

FIGURE 1 | The pedigrees, DNA chromatograms, EEG, and bioinformatical annotations of the E1623A variant of SCN1A gene identified from two cases of epilepsy. (A) Pedigrees of two cases. Case 1 was a 17.5-year-old boy with Dravet syndrome. The proband of case 2 was diagnosed as epilepsy with febrile seizure plus. His mother and elder sister had several febrile seizures before 6 years old. A/E indicates heterozygous E1623A carrier, while E/E indicates wildtype. (B) Sanger sequencing verification. (C) Representative EEG of the case 1 proband showing the generalized high amplitude sharp and slow waves (left), along with focal sharp and slow waves in the left frontal and temporal regions (right). (D) Collection of reference sequences and human variants within the S3-S4 extracellular loop of DIV where the E1623A occurred. The position at E1623 has not been reported with any variant according to human databases. The collected missense variants with epilepsy or non-clinical implications are indicated by red or green bars with their substituted AA abbreviations. Available minor allele frequency ( $f$ value) is under the reference SNP IDs. (E) Structure-function map of $\mathrm{Na}_{v} 1.1$ indicating the location of E1623A. (F) A top view of 3D structural modeling of the Nav1.1 alpha unit shows that the S3-S4 loop and E1623 were at a distance from the central pore region. (G) A side view of the arrangement of the VSD highlights the extracellular S3-S4 loop and the location of E1623. The S4 helices are depicted in pink, while other helices are in green. The S3-S4 loop is highlighted in red.

included alanine (A), aspartic acid (D), glycine $(\mathrm{G})$, lysine $(\mathrm{K})$, glutamine $(\mathrm{Q})$, and valine $(\mathrm{V})$. These residues together with glutamate happen to show a spectrum of residues with different properties, such as molecular mass, polarity, and charge properties (Figure 3).

The WT and diverse mutant alpha-subunits, which were produced by site-directed mutagenesis, were expressed transiently in HEK293T cells. We first examined the plasma membrane expression of these mutants by Western blot and did not find any significant difference among the mutants (Supplementary Data). Subsequently, the mutants were subjected to examine their functional effects by using whole-cell voltage clamp recording. These mutants manifested many different amplitudes of $\mathrm{Na}^{+}$currents (Figure 4A). The peak current density of the epilepsy-associated mutant E1623A was significantly decreased $(P<0.05$; Figures 4B-D), while the mutant E1623Q was slightly increased when compared with that of WT, but did not reach statistical significance $(P>0.05)$. It was noted that E1623K and E1623V also resulted in a significant decrease in current density $(P<0.05)$.

The normalized conductance-voltage ( $\mathrm{G}-\mathrm{V}$, activation) curve of the E1623A showed a slight right shift compared with the WT, with a slower voltage-dependent rising. This is evident as a statistically significant difference in the parameter of 


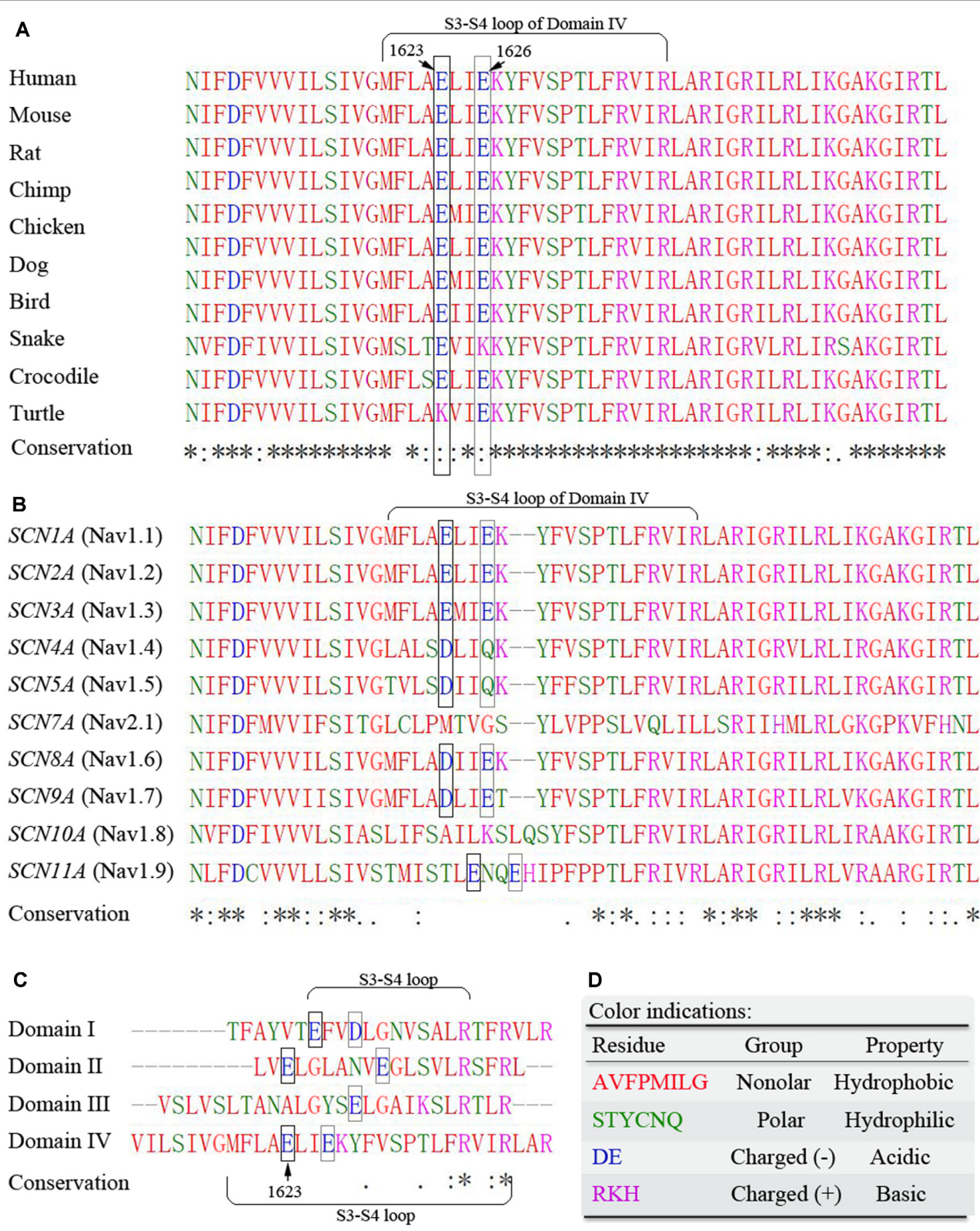

FIGURE 2 | Multiple sequence alignments of the S3-S4 extracellular loop of DIV. (A) Cross-species alignments show high conservation of amino acid sequence within the region. (B) Sequence alignments among $\mathrm{Na}_{\mathrm{v}}$ family members indicate high conservation of two acidic residues E1623 and E1626. (C) Differences in sequence length and residues of the S3-S4 loop among different domains of human Nav 1.1 alpha unit. (D) Color indications. Residues are grouped by their physicochemical properties and indicated by different colors. The symbols of conservation significance are in keeping with the Clustal Omega from the NCBI website. * fully conserved; : conservation with strongly similar properties; . conservation with weakly similar properties; blank space indicates not conserved. The potentially important acidic residues are highlighted with frames.

the slope of steady-state activation between E1623A and WT (Figure 5A). The depolarized voltage dependence of activation may reduce the availability of the E1623A mutant channels to operate as amplifiers of subthreshold depolarization. The mutant E1623V showed similar alteration, and the other mutants caused slight alterations of activation parameters without statistical 


\section{Wildtype: $c .4867-9 \mathrm{GAG} \rightarrow \mathrm{Glu}^{\S}$}

c. $4867 \mathrm{G}>$

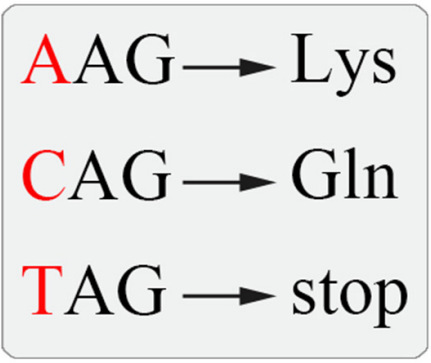

c. $4868 \mathrm{~A}>$
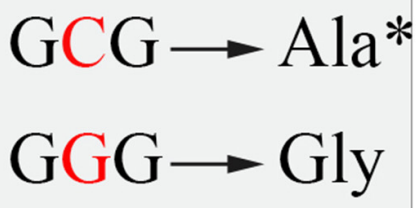

$\mathrm{GTG} \rightarrow \mathrm{Val}$ c. $4869 \mathrm{G}>$

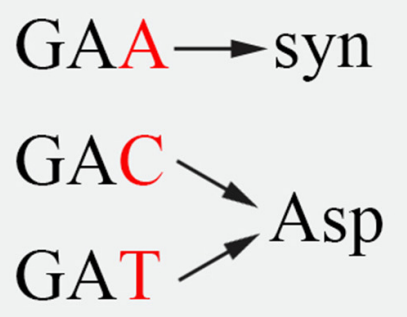

B

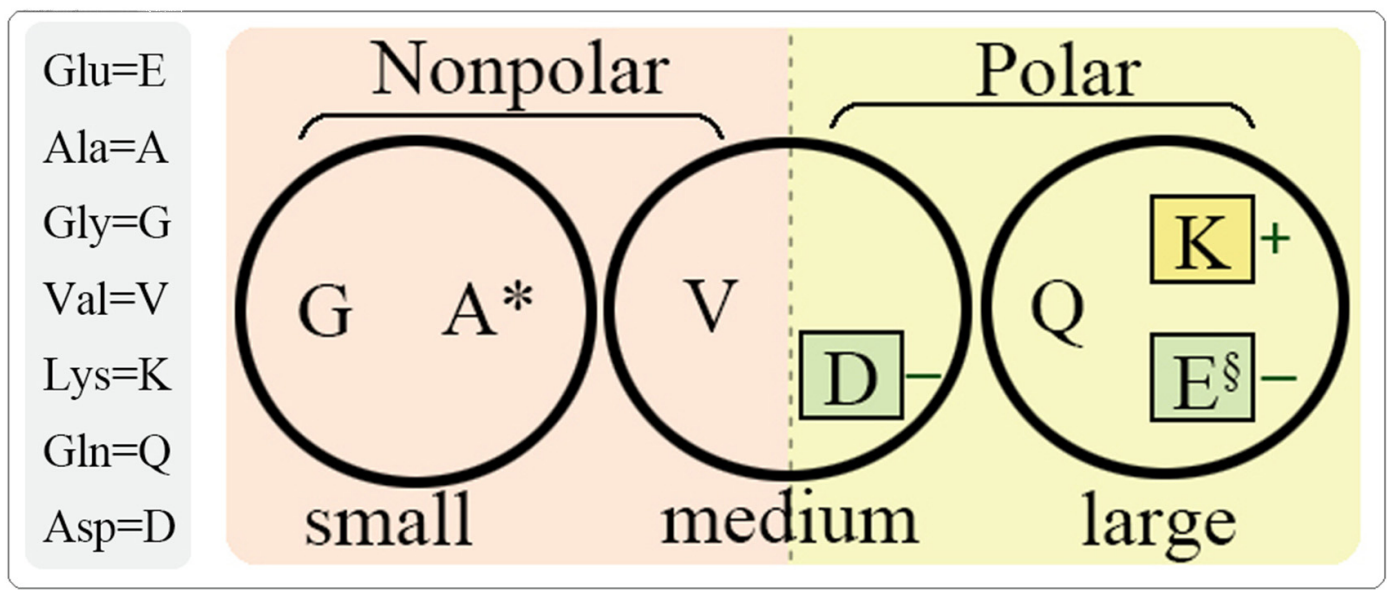

FIGURE 3 | Schematic diagram of site-directed mutagenesis at E1623. (A) All latent variants by single nucleotide variation at C.4867-4869. (B) Residue grouping according to their basic physiochemical properties, including molecular weight (circles), polar (colored squares), and charge (little colored squares). §, wild-type; *, the identified mutation.

significance. Different from a continuity of variations in the steady-state activation curves, the variants were presented as two distinct groups in fast inactivation property (Figure 5B). The steady-state inactivation curves of the mutants E1623A, E1623K, E1623Q, and E1623V were almost overlapped and significantly shifted in the hyperpolarizing direction, whereas the mutants E1623D and E1623G did not differ from the WT. Statistical analysis evidenced that there were two significant subsets on the potential of half-inactivation, but without difference in the slope.

Recovery from inactivation was assessed by using a standard two-pulse protocol. All examined mutants recovered fully within $1 \mathrm{~s}$ in this experiment. The fractional recoveries of $\mathrm{Na}_{\mathrm{v}} 1.1$ peak current were plotted against the intervals on a logarithmic time scale. The WT and mutant channels exhibited significantly different kinetics (Figure 5C). Statistical analysis of the time constants ( $\tau$-fast and $\tau$-slow) revealed that the E1623A mutant channel recovered from inactivation at the slowest rate among the mutants and was exclusively different from the WT. The
E1623A mutant also differed significantly from the E1623D, E1623Q, E1623K, and E1623G mutants in $\tau$-fast.

Taken together, the epilepsy-associated mutant E1623A had significant alterations in all of the four aspects of electrophysiological channel properties and presented the most apparent alteration in three of the four aspects when compared with the other potential mutants. E1623V most closely resembled E1623A, with a reduction in channel conductance and availability during activation and inactivation. E1623Q and E1623K had significant hyperdepolarization shifts only in the voltage-dependent inactivation. No significant alteration was found in either E1623D or E1623G.

\section{Relationship Between Residue Properties and Electrophysiological Parameters}

We analyzed the correlation between amino acid properties and functional alterations of $\mathrm{Na}_{\mathrm{v}} 1.1$ channel (Table 1). The physicochemical properties including molecular size, charge, and 

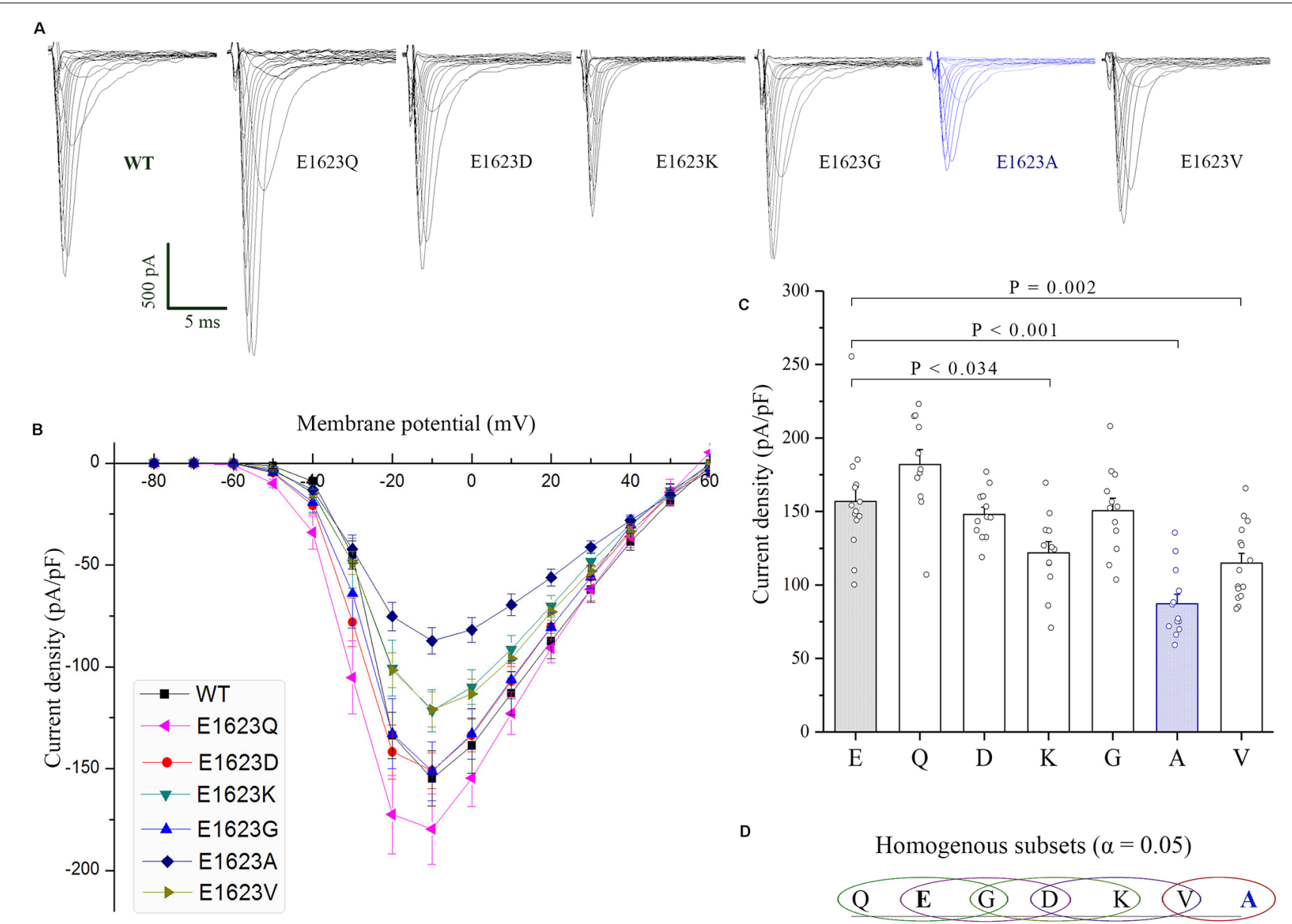

FIGURE 4 | Current densities of E1623 mutants. All variants were expressed in HEK293T cells via transfection. (A) Representative current traces elicited with 20 -ms depolarizations ranging from $-80 \mathrm{mV}$ to $+60 \mathrm{mV}$ in $+10 \mathrm{mV}$ steps. Intervals between pulses were $10 \mathrm{~s}$. The mutants were arrayed by their residue similarity to WT according to Grantham's distance. Currents are noticeably reduced in the E1623A variant. (B) Plots of current densities and membrane potentials. (C) Group data of peak current densities elicited at $-10 \mathrm{mV}$. (D) Statistical output of homogenous subsets shows significant differences at the 0.05 level among the variants, via one-way ANOVA and post hoc Tukey HSD tests.

polarity were parameterized by their mass, isoelectric point, and hydrophobicity scale, respectively (Supplementary Data); and the ensemble molecular difference was assessed by the established matrices. As shown in Table 1, the hydrophilicity scale was significantly correlated with the channel voltagedependent activation (slope of activation, $r=0.770, P<0.05$ ) and the recovery (slow recovery, $r=0.870, P<0.05$ ) parameters. We did not find any significant contributor to current density, which is closely related to the pathogenicity of the mutants. No quantitative correlation between residue properties and inactivation was found, either (Table 1).

We analyzed correlations between the predictive scores and alteration of current density. Absolute changes in average current density of the six variants at E1623 were plotted against the normalized predictive scores obtained by different in silico tools, including evolutionary conservation-based methods (e.g., SIFT and Mutationassessor), structure-based (protein stability) predicting tools (e.g., Strum and I-mutant suite), and machinelearning methods that combined comprehensive information (e.g., SNAP2 and PROVEAN; Figure 6). The predictive scores from SNAP2 and PROVEAN appeared to be matched better than other predictive scores ( $r=0.486$ and $r=0.485$, respectively). However, these predictive scores did not correlate well with the alterations in current density in general.

\section{DISCUSSION}

The sodium channel plays a critical role in controlling the excitability of cells. $\mathrm{Na}_{\mathrm{v}} 1.1$ appears to be a particularly important molecule since it is frequently associated with human diseases (Meisler and Kearney, 2005). Our previous study shows that $\mathrm{Na}_{\mathrm{v}} 1.1$ is highly sensitive to genetic variations (Chen et al., 2015) and thus may be used as a model or an example to explore molecular bases underlying the functioning of ion channels. The present study identified a novel epilepsy-associated mutation E1623A within the extracellular S3-S4 loop of DIV, a previously unidentified functional component. Functional studies demonstrated remarkable loss- 
A

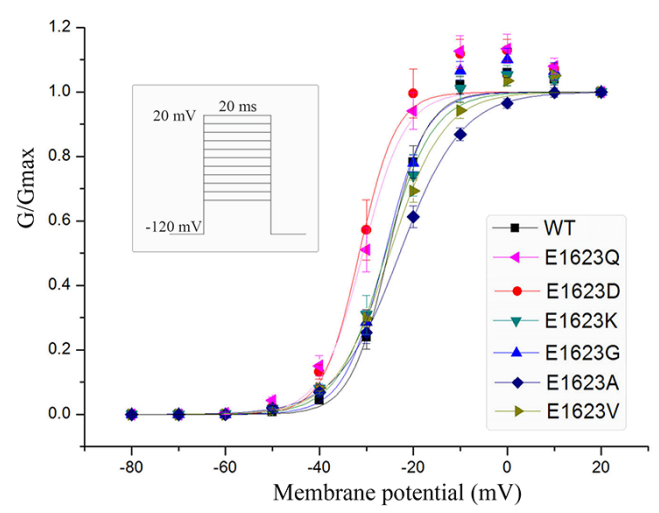

B

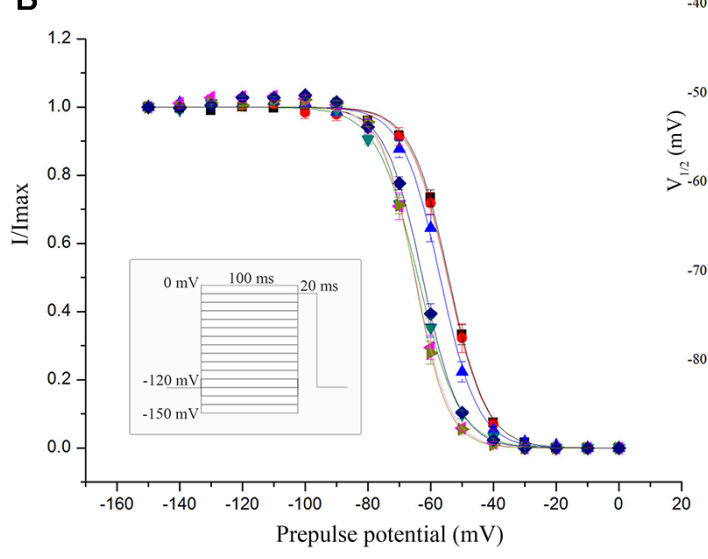

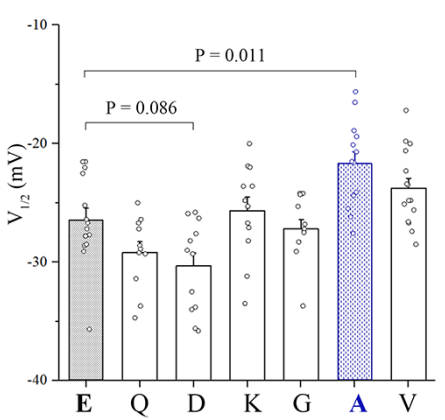
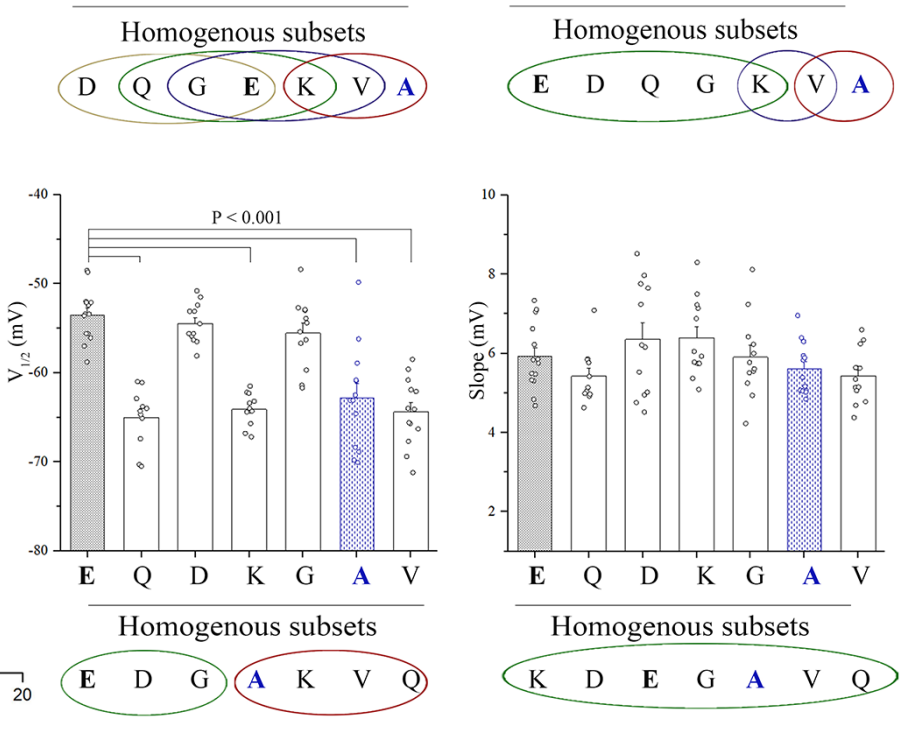
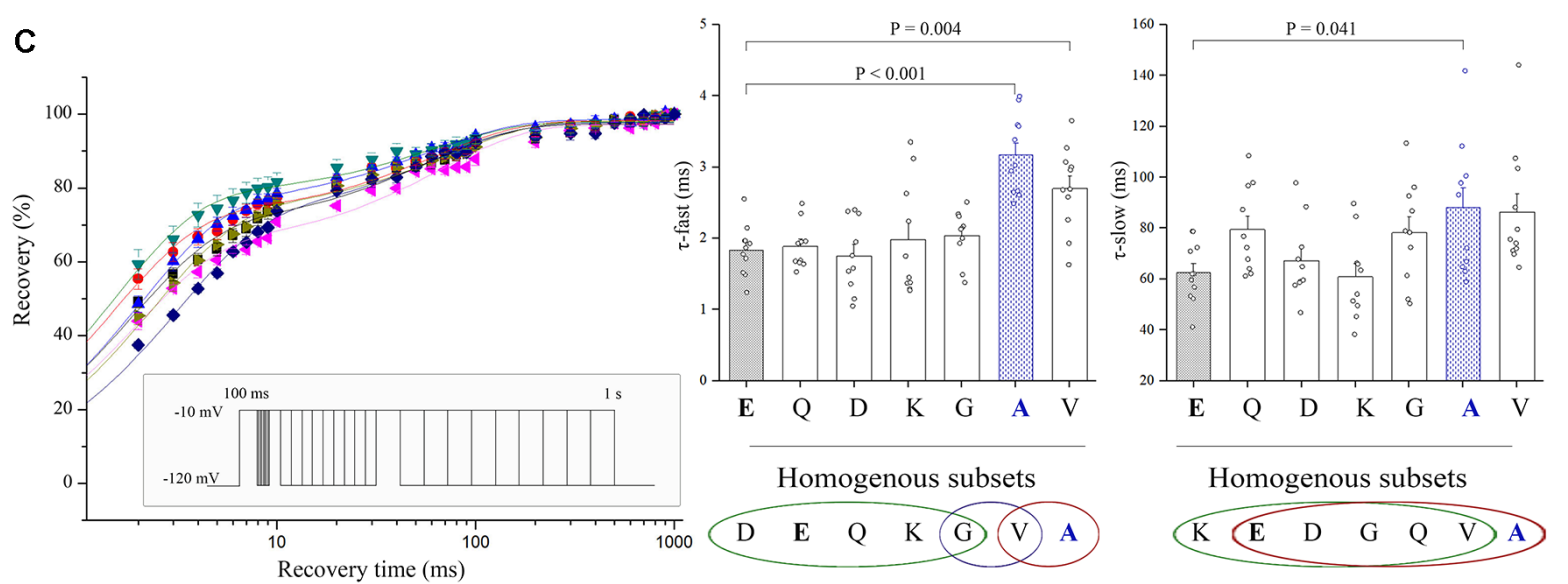

FIGURE 5 | Voltage dependence and recovery kinetics of the E1623 mutants. (A) Activation curves of all E1623 mutants. (B) Steady-state inactivation curves. For testing voltage dependent channel availability in fast inactivation, a two-pulse protocol was used. Na ${ }^{+}$currents were evoked by a 20 -ms test pulse to - 10 m $V$ after 100-ms conditioning prepulses between -150 and $0 \mathrm{mV}$ in 10-mV increments. Peak currents evoked by test pulses were measured, normalized, and plotted against the conditioning prepulse potentials. Parameters of activation and fast inactivation were estimated by fitting current-voltage relationships with the Boltzmann function. Estimated parameters including half-activation potential $\left(V_{1 / 2}\right)$ and slope factor, were derived from the Boltzmann fits. Group data of the $V_{1 / 2}$ and slope are shown at the right panels. (C) Recovery from fast inactivation in paired stimulus experiments. Paired stimuli were applied at various inter-stimulation intervals. The recovery fractions were plotted to the intervals. The normalized recovery plots were fit with a double-exponential function to obtain parameters of $\tau$-fast and $\tau$-slow (shown in right panels). Statistical outputs of homogenous subsets (shown below the plots; those within the same circle are $\alpha>0.05$ ) indicate significant differences among the mutants.

of-function of E1623A mutant, with characteristic alterations in activation, inactivation, and recovery properties, and confirmed the pathogenicity of E1623A. More importantly, the other substitutions at the residue E1623 also resulted in apparent 
TABLE 1 | Correlation coefficients between residue similarity and functional changes.

\begin{tabular}{|c|c|c|c|c|c|}
\hline \multirow[b]{2}{*}{ Indices for AA differences } & \multirow[b]{2}{*}{$\begin{array}{l}\text { Conductance } \\
\text { Peak current }\end{array}$} & \multirow[b]{2}{*}{$\begin{array}{l}\text { Activation } \\
\text { slope }\end{array}$} & \multirow[b]{2}{*}{$\begin{array}{c}\text { Inactivation } \\
V_{1 / 2}\end{array}$} & \multicolumn{2}{|c|}{ Recovery } \\
\hline & & & & $\tau$-fast & $\tau$-slow \\
\hline \multicolumn{6}{|l|}{ Simple property } \\
\hline Volume & 0.114 & 0.394 & -0.376 & 0.544 & 0.353 \\
\hline Charge & 0.361 & 0.274 & 0.556 & 0.018 & -0.140 \\
\hline Polarity & 0.482 & $0.770^{*}$ & 0.290 & 0.705 & $0.870^{\star}$ \\
\hline \multicolumn{6}{|l|}{ Physicochemical distance } \\
\hline Miyata's distance & 0.258 & 0.599 & 0.039 & 0.577 & 0.626 \\
\hline Grantham's distance & 0.392 & 0.720 & 0.083 & 0.688 & 0.730 \\
\hline Sneath's index & 0.406 & 0.634 & 0.198 & 0.554 & 0.413 \\
\hline Experimental exchangeability & 0.079 & 0.140 & -0.654 & 0.439 & 0.249 \\
\hline
\end{tabular}

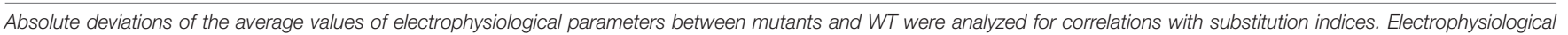

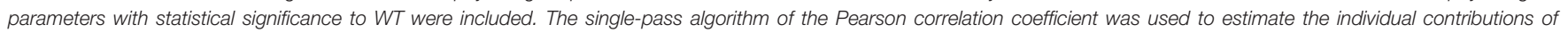

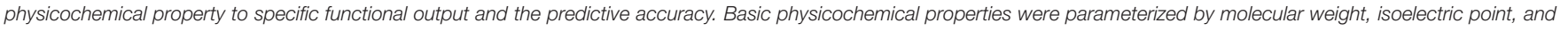
hydrophobicity. The hydrophobicity scale of Kyte and Doolittle was adopted as the values of hydrophobicity (Kyte and Doolittle, 1982$)$. *Significant correlation, $P<0.05$.

functional alterations, suggesting that the residue E1623, presumably as well as the adjacent residues within the extracellular loop, play a critical role in channel gating.

Previous studies have demonstrated that missense mutations in the central pore region of $\mathrm{Na}_{\mathrm{V}} 1.1$ were exclusively associated with loss-of-function, while mutations in the other regions were associated with diverse functional alterations (Meng et al., 2015). In the present study, the novel epilepsy-associated mutation E1623A is located at the extracellular loop linking S3 and S4 in DIV. The roles of extracellular components, especially the loops that connect the transmembrane segments, in the function of $\mathrm{Na}_{\mathrm{v}} 1.1$ channel complex are largely unknown. Based on topological modeling, this extracellular S3-S4 loop appears to be at a considerable distance from the central pore, and is theoretically unlikely to result in a functional change. However, both the severe clinical phenotype in our cases and the existing clinical variants within the loop indicated that the E1623 might be critical for proper channel function.

The subsequent functional studies revealed that the E1623A variant resulted in surprising abnormalities in gating properties, including channel conductance and gating kinetics. Several other potential missense mutants at E1623 also led to changes in gating properties, although to a lesser extent. It is quite interesting that the substitutions in a residue at the extracellular loop that is far apart from the central pore, lead to an overwhelming alteration in channel gating. Since E1623 is located a distance away from the central pore and appears not directly involved in ion conduction, other unknown mechanisms such as electrostatic interaction and local conformational flexibility may be involved. It has been established that sodium currents are elicited in response to changes in the membrane potential sensed by the channel voltage sensor module (S1-S4) containing the S3-S4 linker. Recent studies have uncovered intricate interactions of these elements within the $\mathrm{Na}_{\mathrm{v}}$ channel signaling complex (Okamura et al., 2015; Catterall et al., 2017). The positive charges in the S4 segments serve as gating charges and move across the membrane electric field when activated, initiating conformational changes to open and close the channel (Yarov-Yarovoy et al., 2012). Upon opening, the upper part of the S4 approaches the pore domain.
The S3-S4 loop is lying on the top of the S1-S4 bundles, extending into the extracellular space (Figure 1). Such location suggests that S3-S4 loops may confer local conformational flexibility and thereby impact channel functioning. In a previous study on small-conductance calcium-dependent potassium, a 3-amino acid motif within the extracellular S3-S4 loop was suggested to determine apamin blocking effects and regulate the allosteric change of gating pore (Weatherall et al., 2011). Here multiple sequence alignments of the S3-S4 loop in DIV showed a conserved residue cluster of negative charged residues glutamate $\left(\mathrm{E}_{\mathrm{XX}} \mathrm{E}\right)$ among $\mathrm{Na}_{\mathrm{v}}$ superfamily members and negatively charged residues glutamate or aspartate (ExxE/DxxE) in other voltage-gated sodium channels (Figure 2). A similar sequence is present in the S3-S4 loops of different domains. The ExxE forms the tight turn of the loop (Figure 1) and is located close to the voltage-sensor. Therefore, the ExxE motif and the negatively charged acidic glutamate may be crucial for channel functioning, implying that the molecular sub-regional effect should be considered in evaluating the pathogenicity of variants.

It has been demonstrated that mutations of charged residues in S4 of all domains affected activation, whereas those in S4 segments of primarily DI and DIV had the most effects on fast inactivation (Groome and Winston, 2013). Similarly, charge-reversing mutations in S1-S3 segments alter sodium channel activation and fast inactivation, suggesting that positive charge movement across the bilayer must be facilitated by negative charges in other parts of the protein (Chowdhury and Chanda, 2012). In the voltage-gated potassium channels, similar conserved negatively charged residues in VSD have been shown to actively catalyze the transport of ionized groups through the cell membrane and control the voltage sensor operation (Starace and Bezanilla, 2004; Pless et al., 2011; Lacroix et al., 2014). These negatively charged residues are proposed to act as neutralization and electrostatic interactions with positively charged S4 residues, and thus have profound effects on activation gating (Papazian et al., 1995; Yang et al., 2009). Previously, no experimental evidence has been obtained on the role of the extracellular S3-S4 loop in sodium channel functioning. In 
A
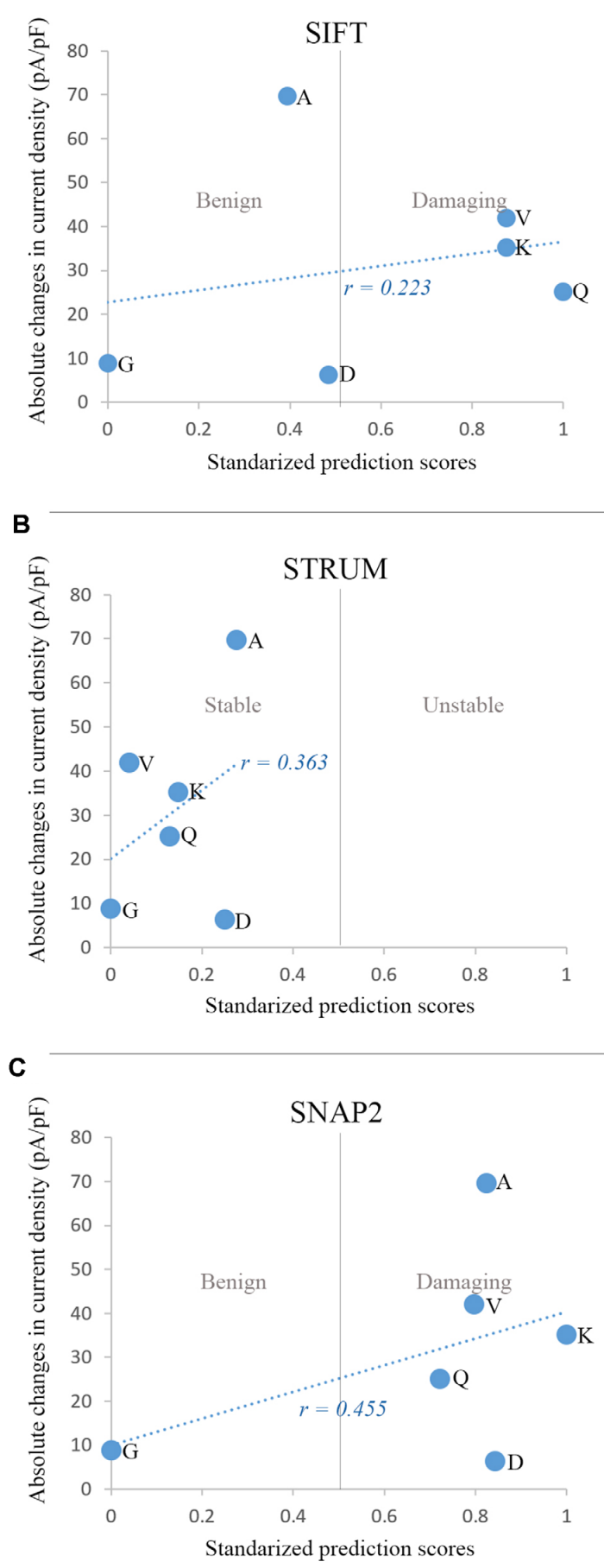
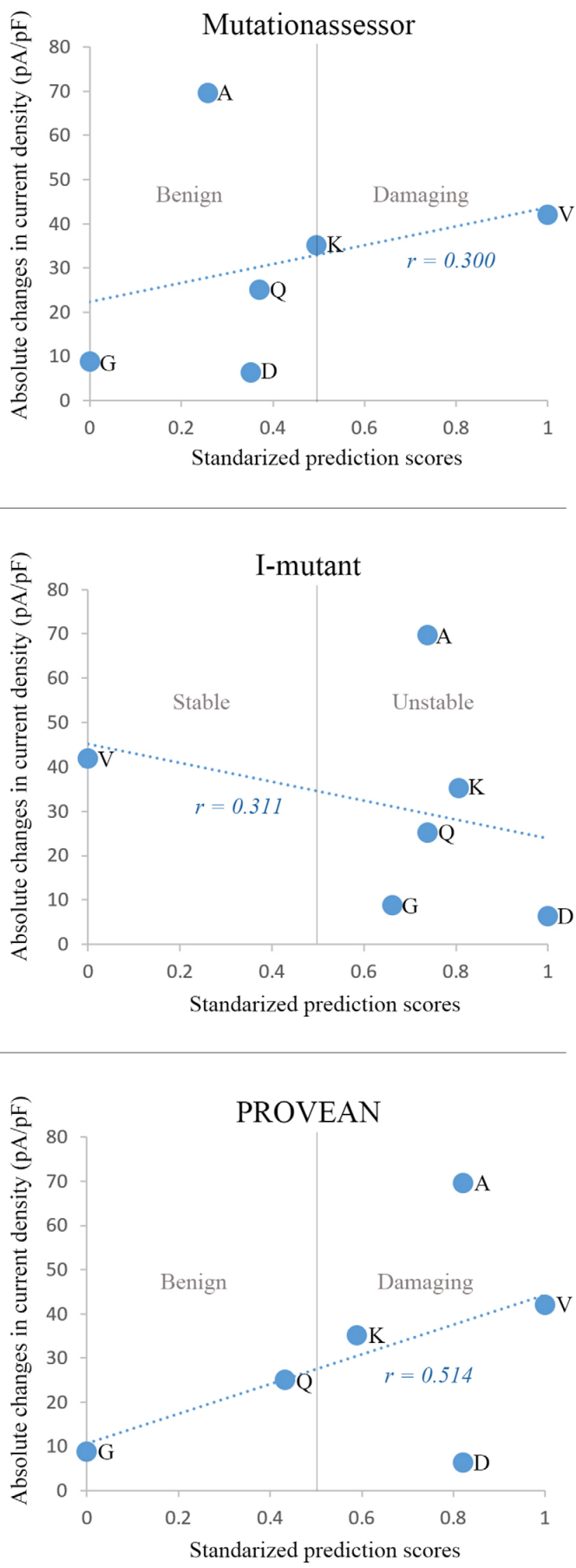

FIGURE 6 | Correlations between in silico prediction scores and functional changes in current density. (A) Sequence and evolutionary conservation-based methods: SIFT and Mutationassessor. (B) Prediction of protein stability changes by Strum and I-mutant suite. (C) Machine-learning methods combining comprehensive features: SNAP2 and PROVEAN. For comparison, prediction scores of the six variants from different tools were normalized to the Polyphen-2 scoring scheme, with a range from 0 to 1 and a threshold at 0.5 . The minimal score with benign prediction was set as 0 , and the maximal score with damaging prediction was set as 1 . Scores with benign prediction were scaled to a range from 0 to 0.5 , while scores of damaging variants were scaled to a range from 0.5 to 1.

the present study, the charge-reversing mutation E1623K led to significant changes in fast inactivation with a shifting of steady-state inactivation to more hyperpolarized potentials. In contrast, the negative charge-retaining mutant E1623D showed no alteration in inactivation and was mostly close to WT in fast inactivation parameters (Figure 5). These findings suggest 
that the negative charge property of E1623 is critical in the inactivation of the sodium channel. Note that the sequence in the N-terminal part of the loop just after S3 is MFLAEL. The residues $\mathrm{M}, \mathrm{L}$, and A have high helix propensities. Replacing the E with an A may change the helix-loop-helix structure, and the S3 helical extension into the S3-S4 loop is quite likely to have some effects on the voltage-responsiveness of the S4 helix that could affect both channel activation and inactivation as observed experimentally.

Several mutants without charge-reversing or helix transformation also demonstrated apparent effects on channel activation/inactivation, suggesting much complex relationships between the residue properties of the extracellular loop and channel functioning. We analyzed the correlations between residue properties and electrophysiological alterations. Significant correlations were detected between residue polarity and channel properties (Table 1), indicating that hydrophobicity at the residue E1623 of the extracellular loop is a key property affecting channel functioning. Hydrophobic/polar property is a meaningful scheme clustering residue alphabets. The importance of hydrophobic interaction in protein structure owes much to the following two facts: (i) it is the driving force for protein folding (Dill, 1990); and (ii) it is an important factor for proteinprotein interaction (Jones and Thornton, 1996). For hydrophilic glutamate at the extracellular site and on the top of VSDs, its hydrophilic property may play an additional role in favoring the concentration and flow of charged $\mathrm{Na}^{+}$through the lipid bilayer, because extracellular $\mathrm{Na}^{+}$is conducted as a hydrated ion (Payandeh et al., 2012).

However, we did not find any remarkable correlations between the residue properties and peak current density that reflected channel conductance. The present study revealed that the hydrophilic glutamate residue substituted by hydrophobic residues at E1623, such as E1623V and E1623A, did result in decreased current density. However, there were also polarityaltered mutants that did not affect current density, such as E1623G and E1623Q. The confounding factors for the channel conductance at E1623 may include phylogenetic and structural context, together with the elementary changes in amino acid properties. To explore the factors that contributed to channel dysfunction, we used in silico tools to score the mutants from three different perspectives, including sequence/evolutionary conservation, protein stability, and machine-learning protocol with combined comprehensive information and variant dataset of known pathogenicity, based on their working principles. The machine-learning based predictors, SNAP2 and PROVEAN, showed a relatively higher correlation coefficient between the predictive scores and channel conductance.

The conductance of $\mathrm{Na}_{\mathrm{v}} 1.1$ indicated by peak current density is the most crucial property that determines macroscopic gain or loss of channel function. The common form of functional defects caused by genetic variants, identified in epilepsy patients, is loss of function or partial loss of function, featured by non-detectable or reduced sodium current in electrophysiological recordings. Changes in channel conductance determine the pathogenicity of mutations (Tang et al., 2020). In contrast, the electrophysiological parameters of voltage-dependent kinetics of the channel determine the channel characteristics and are associated with mild clinical phenotypes (Liao et al., 2010; Meng et al., 2015). Understanding what residues and functional domains would associate with channel conductance and gating kinetics would provide novel insights into the molecular basis of channel functioning and pathogenicity of variants in channels.

Theoretically, one residue is potentially subject to several different substitutions and subsequently associated with variations in channel function and phenotype, which brings challenges in clinical recognition and diagnosis of genetic disorders. The present study explored the correlation between the nature of substitution and functional alteration in sodium channel $\mathrm{Na}_{\mathrm{v}}$ 1.1. It would help understand the intrinsic mechanism underlying the pathogenicity of missense variants. Under the influence of some extrinsic factors, individual cases might exhibit diversity in clinical phenotype and inheritance. In our example, the case 1 and case 2 presented different epileptic phenotypes and inheritance, in which one was severe DS and de novo and the other was epilepsy with febrile seizure plus and dominantly inherited from his mother. In general, a vast majority of de novo severe mutations, especially truncations, were identified in patients with DS, while missense mutations were responsible for familial cases with milder phenotypes (Meng et al., 2015; Liu et al., 2018). This is because de novo mutations are unlikely to pass down to the offspring in patients with severe phenotypes like DS. However, the genotypephenotype correlation is not entirely consistent. Under some circumstances, the DS-associated mutations, such as the E1623A and a previously reported p.Arg1912X truncation (Jaimes et al., 2020), might exhibit milder phenotype, and thus have the opportunity to develop into an inherited disease in pedigree. The variable disease expressivity might come from individual differences from the modulation of channel physiology, such as trafficking, posttranslational modifications, and pharmacological modulators. It remains a challenging conundrum for researchers about how biophysical alterations in a mutant $\mathrm{Na}_{\mathrm{v}} 1.1$ determine their phenotypes.

Although there has been great progress in genetic sequencing and in silico prediction, variant interpretation remains a major challenge. The present study proposed not only an essential role of the extracellular S3-S4 loop at domain IV in channel functioning, but also an urge of customized predictive tools with more considerations, including 3D structure information, experimental data, and sub-regional function, etc. Different channel functional properties, such as channel conductance and voltage-dependent kinetics, are potentially affected by the distinct molecular alterations and associated with different clinical phenotypes, providing new perspectives on molecular mechanisms underlying genetic diseases.

\section{DATA AVAILABILITY STATEMENT}

The original contributions presented in the study are included in the article/Supplementary Materials, further inquiries can be directed to the corresponding author. 


\section{ETHICS STATEMENT}

The studies involving human participants were reviewed and approved by Ethics Committee of the Second Affiliated Hospital of Guangzhou Medical University. Written informed consent to participate in this study was provided by the participants' legal guardian/next of kin. The animal study was reviewed and approved by Ethics Committee of the Second Affiliated Hospital of Guangzhou Medical University. Written informed consent was obtained from the individual(s), and minor(s)' legal guardian/next of kin, for the publication of any potentially identifiable images or data included in this article.

\section{AUTHOR CONTRIBUTIONS}

W-PL performed the conceptualization and funding acquisition. W-PL, TS, and M-LC designed research. TS, M-LC, HM, LHL, BT, X-RL, and W-PL performed research and/or analyzed data. TS wrote the manuscript draft. W-PL contributed to critical manuscript revisions. All authors contributed to the article and approved the submitted version.

\section{REFERENCES}

Bosmans, F., Martin-Eauclaire, M. F., and Swartz, K. J. (2008). Deconstructing voltage sensor function and pharmacology in sodium channels. Nature 456, 202-208. doi: 10.1038/nature07473

Catterall, W. A., Wisedchaisri, G., and Zheng, N. (2017). The chemical basis for electrical signaling. Nat. Chem. Biol. 13, 455-463. doi: 10.1038/nchembio.2353

Ceulemans, B. P., Claes, L. R., and Lagae, L. G. (2004). Clinical correlations of mutations in the SCN1A gene: from febrile seizures to severe myoclonic epilepsy in infancy. Pediatr. Neurol. 30, 236-243. doi: 10.1016/j.pediatrneurol. 2003.10.012

Chen, Y. J., Shi, Y. W., Xu, H. Q., Chen, M. L., Gao, M. M., Sun, W. W., et al. (2015). Electrophysiological differences between the same pore region mutation in SCN1A and SCN3A. Mol. Neurobiol. 51, 1263-1270. doi: 10.1007/s12035-0148802-x

Chowdhury, S., and Chanda, B. (2012). Perspectives on: conformational coupling in ion channels: thermodynamics of electromechanical coupling in voltagegated ion channels. J. Gen. Physiol. 140, 613-623. doi: 10.1085/jgp.201210840

Claes, L., Ceulemans, B., Audenaert, D., Smets, K., Lofgren, A., Del-Favero, J., et al. (2003). De novo SCN1A mutations are a major cause of severe myoclonic epilepsy of infancy. Hum. Mutat. 21, 615-621. doi: 10.1002/humu.10217

den Dunnen, J. T., and Antonarakis, S. E. (2000). Mutation nomenclature extensions and suggestions to describe complex mutations: a discussion. Hum. Mutat. 15, 7-12. doi: 10.1002/(SICI)1098-1004(200001)15:1<7::AIDHUMU4>3.0.CO;2-N

Dill, K. A. (1990). Dominant forces in protein folding. Biochemistry 29, 7133-7155. doi: $10.1021 /$ bi00483a001

Dravet, C., and Oguni, H. (2013). Dravet syndrome (severe myoclonic epilepsy in infancy). Handb. Clin. Neurol. 111, 627-633. doi: 10.1016/b978-0-444-52891-9. 00065-8

Gambardella, A., and Marini, C. (2009). Clinical spectrum of SCN1A mutations. Epilepsia 50, 20-23. doi: 10.1111/j.1528-1167.2009.02115.x

Gataullina, S., and Dulac, O. (2017). From genotype to phenotype in Dravet disease. Seizure 44, 58-64. doi: 10.1016/j.seizure.2016.10.014

Groome, J. R., and Winston, V. (2013). S1-S3 counter charges in the voltage sensor module of a mammalian sodium channel regulate fast inactivation. J. Gen. Physiol. 141, 601-618. doi: 10.1085/jgp.201210935

Harkin, L. A., McMahon, J. M., Iona, X., Dibbens, L., Pelekanos, J. T., Zuberi, S. M., et al. (2007). The spectrum of SCN1A-related infantile epileptic encephalopathies. Brain 130, 843-852. doi: 10.1093/brain/awm002

\section{FUNDING}

This work was supported by the National Natural Science Foundation of China (grant No. 81571273 and 81271197), the Science and Technology Project of Guangdong Province (grant No. 2017B09090436 and 2017B030314159), and Science and Technology Project of Guangzhou (grant No. 201904020028). The funders had no role in study design, data collection and analysis, and decision to publish or preparation of the manuscript.

\section{ACKNOWLEDGMENTS}

We are deeply grateful to the patients and clinicians who participated in this study.

\section{SUPPLEMENTARY MATERIALS}

The Supplementary Material for this article can be found online at: https://www.frontiersin.org/articles/10.3389/fnmol.2021. 797628/full\#supplementary-material.

He, N., Lin, Z. J., Wang, J., Wei, F., Meng, H., Liu, X. R., et al. (2019). Evaluating the pathogenic potential of genes with de novo variants in epileptic encephalopathies. Genet. Med. 21, 17-27. doi: 10.1038/s41436-018-0011-y

Jaimes, A., Guerrero-Lopez, R., Gonzalez-Giraldez, B., and Serratosa, J. M. (2020). De novo truncating mutation in SCN1A as a cause of febrile seizures plus (FS+). Epileptic. Disord. 22, 323-326. doi: 10.1684/epd.2020.1167

Jones, S., and Thornton, J. M. (1996). Principles of protein-protein interactions. Proc. Natl. Acad. Sci. U S A 93, 13-20. doi: 10.1073/pnas.93.1.13

Kasperaviciute, D., Catarino, C. B., Matarin, M., Leu, C., Novy, J., Tostevin, A., et al. (2013). Epilepsy, hippocampal sclerosis and febrile seizures linked by common genetic variation around SCN1A. Brain 136, 3140-3150. doi: 10.1093/brain/awt233

Kyte, J., and Doolittle, R. F. (1982). A simple method for displaying the hydropathic character of a protein. J. Mol. Biol. 157, 105-132. doi: 10.1016/0022-2836(82)90515-0

Lacroix, J. J., Hyde, H. C., Campos, F. V., and Bezanilla, F. (2014). Moving gating charges through the gating pore in a $\mathrm{Kv}$ channel voltage sensor. Proc. Natl. Acad. Sci. U S A 111, E1950-E1959. doi: 10.1073/pnas.140616 1111

Liao, W. P., Shi, Y. W., Long, Y. S., Zeng, Y., Li, T., Yu, M. J., et al. (2010). Partial epilepsy with antecedent febrile seizures and seizure aggravation by antiepileptic drugs: associated with loss of function of $\mathrm{Na}(\mathrm{v})$ 1.1. Epilepsia 51, 1669-1678. doi: 10.1111/j.1528-1167.2010.02645.x

Liu, J., Tong, L., Song, S., Niu, Y., Li, J., Wu, X., et al. (2018). Novel and de novo mutations in pediatric refractory epilepsy. Mol. Brain 11:48. doi: 10.1186/s13041-018-0392-5

Lossin, C., Rhodes, T. H., Desai, R. R., Vanoye, C. G., Wang, D., Carniciu, S., et al. (2003). Epilepsy-associated dysfunction in the voltage-gated neuronal sodium channel SCN1A. J. Neurosci. 23, 11289-11295. doi: 10.1523/JNEUROSCI.2336-11289.2003

Meisler, M. H., and Kearney, J. A. (2005). Sodium channel mutations in epilepsy and other neurological disorders. J. Clin. Invest. 115, 2010-2017. doi: 10.1172/JCI25466

Meng, H., Xu, H. Q., Yu, L., Lin, G. W., He, N., Su, T., et al. (2015). The SCN1A mutation database: updating information and analysis of the relationships among genotype, functional alteration and phenotype. Hum. Mutat. 36, 573-580. doi: 10.1002/humu.22782

Mulley, J. C., Scheffer, I. E., Petrou, S., Dibbens, L. M., Berkovic, S. F., and Harkin, L. A. (2005). SCN1A mutations and epilepsy. Hum. Mutat. 25, 535-542. doi: 10.1002/humu.20178 
Nabbout, R., Gennaro, E., Dalla Bernardina, B., Dulac, O., Madia, F., Bertini, E., et al. (2003). Spectrum of SCN1A mutations in severe myoclonic epilepsy of infancy. Neurology 60, 1961-1967. doi: 10.1212/01.wnl.0000069463.41870.2f

Okamura, Y., Fujiwara, Y., and Sakata, S. (2015). Gating mechanisms of voltage-gated proton channels. Annu. Rev. Biochem. 84, 685-709. doi: 10.1146/annurev-biochem-060614-034307

Pan, X., Li, Z., Jin, X., Zhao, Y., Huang, G., Huang, X., et al. (2021). Comparative structural analysis of human Nav1.1 and Nav1.5 reveals mutational hotspots for sodium channelopathies. Proc. Natl. Acad. Sci. U S A 118:e2100066118. doi: 10.1073/pnas.2100066118

Papazian, D. M., Shao, X. M., Seoh, S. A., Mock, A. F., Huang, Y., and Wainstock, D. H. (1995). Electrostatic interactions of S4 voltage sensor in shaker K+ channel. Neuron 14, 1293-1301. doi: 10.1016/0896-6273(95)90276-7

Payandeh, J., Gamal El-Din, T. M., Scheuer, T., Zheng, N., and Catterall, W. A. (2012). Crystal structure of a voltage-gated sodium channel in two potentially inactivated states. Nature 486, 135-139. doi: 10.1038/nature11077

Pless, S. A., Galpin, J. D., Niciforovic, A. P., and Ahern, C. A. (2011). Contributions of counter-charge in a potassium channel voltage-sensor domain. Nat. Chem. Biol. 7, 617-623. doi: 10.1038/nchembio.622

Rogers, J. C., Qu, Y., Tanada, T. N., Scheuer, T., and Catterall, W. A. (1996). Molecular determinants of high affinity binding of alpha-scorpion toxin and sea anemone toxin in the S3-S4 extracellular loop in domain IV of the $\mathrm{Na}+$ channel alpha subunit. J. Biol. Chem. 271, 15950-15962. doi: 10.1074/jbc.271. 27.15950

Starace, D. M., and Bezanilla, F. (2004). A proton pore in a potassium channel voltage sensor reveals a focused electric field. Nature 427, 548-553. doi: 10.1038/nature02270

Tang, B., Li, B., Gao, L. D., He, N., Liu, X. R., Long, Y. S., et al. (2020). Optimization of in silico tools for predicting genetic variants: individualizing for genes with molecular sub-regional stratification. Brief Bioinform. 21, 1776-1786. doi: 10.1093/bib/bbz115

Wang, J., Yarov-Yarovoy, V., Kahn, R., Gordon, D., Gurevitz, M., Scheuer, T., et al. (2011). Mapping the receptor site for alpha-scorpion toxins on a $\mathrm{Na}+$ channel voltage sensor. Proc. Natl. Acad. Sci. U S A 108, 15426-15431. doi: 10.1073/pnas.1112320108

Weatherall, K. L., Seutin, V., Liegeois, J. F., and Marrion, N. V. (2011). Crucial role of a shared extracellular loop in apamin sensitivity and maintenance of pore shape of small-conductance calcium-activated potassium (SK) channels. Proc. Natl. Acad. Sci. U S A 108, 18494-18499. doi: 10.1073/pnas.11107 24108

Yang, Y. C., Hsieh, J. Y., and Kuo, C. C. (2009). The external pore loop interacts with S6 and S3-S4 linker in domain 4 to assume an essential role in gating control and anticonvulsant action in the $\mathrm{Na}(+)$ channel. J. Gen. Physiol. 134, 95-113. doi: 10.1085/jgp.200810158

Yarov-Yarovoy, V., DeCaen, P. G., Westenbroek, R. E., Pan, C. Y., Scheuer, T., Baker, D., et al. (2012). Structural basis for gating charge movement in the voltage sensor of a sodium channel. Proc. Natl. Acad. Sci. U S A 109, E93-102. doi: 10.1073/pnas.1118434109

Conflict of Interest: The authors declare that the research was conducted in the absence of any commercial or financial relationships that could be construed as a potential conflict of interest.

Publisher's Note: All claims expressed in this article are solely those of the authors and do not necessarily represent those of their affiliated organizations, or those of the publisher, the editors and the reviewers. Any product that may be evaluated in this article, or claim that may be made by its manufacturer, is not guaranteed or endorsed by the publisher.

Copyright (C) 2022 Su, Chen, Liu, Meng, Tang, Liu and Liao. This is an open-access article distributed under the terms of the Creative Commons Attribution License (CC BY). The use, distribution or reproduction in other forums is permitted, provided the original author(s) and the copyright owner(s) are credited and that the original publication in this journal is cited, in accordance with accepted academic practice. No use, distribution or reproduction is permitted which does not comply with these terms. 